\title{
MICROARCSEC ASTROMETRY: THE GAIA MISSION
}

\author{
L. LINDEGREN \\ Lund Observatory \\ Box 43, SE-22100 Lund, Sweden \\ AND \\ M.A.C. PERRYMAN \\ Astrophysics Division, ESTEC \\ 2200AG Noordwijk, The Netherlands
}

\section{The GAIA Concept}

The Hipparcos mission demonstrated the efficiency of space astrometry (in terms of number of objects, accuracy, and uniformity of results) and the fact that a relatively small instrument can have a very large scientific potential in the area of astrometry. However, Hipparcos could probe less than 0.1 per cent of the volume of the Galaxy by direct distance measurements. Using a larger instrument and more efficient detectors, it is now technically feasible to increase the efficiency of a space astrometry mission by several orders of magnitude, thus encompassing a large part of the Galaxy within its horizon for accurate determination of parallaxes and transverse velocities. Such a mission will have immediate and profound impact in the areas of the physics and evolution of individual stars and of the Galaxy as a whole.

The Global Astrometric Interferometer for Astrophysics (GAIA) is studied as a possible Cornerstone Mission within the European Space Agency's scientific programme Horizon 2000 Plus. Consisting of up to four optical Fizeau interferometers contained in a single payload and operated by continuous scanning, its target accuracy is about 10 microarcsec or better for the positions, parallaxes and annual proper motions of some 50 million objects down to $V=15$ mag, with many more fainter objects measured at a correspondingly reduced accuracy. Direct recording of the interference fringes by CCDs operated in drift-scan mode will give a limiting magnitude of $V \sim 20$ and capability to observe in crowded regions. Real-time detection of all point sources to well-defined limits may eliminate the need for an input catalogue and result in the observation of up to $10^{9}$ stars, quasars and minor planets. A mission lifetime of 5 years is targeted.

Each Fizeau interferometer has a modest $(<3 \mathrm{~m})$ baseline, a large $\left(\sim 1^{\circ}\right)$ field of view, a wide coverage of visual to near-IR wavelengths $(\lambda \sim 400-1000 \mathrm{~nm})$, and a collecting area of about $0.5 \mathrm{~m}^{2}$. The field of view is covered by a mosaic of CCDs simultaneously recording the fringe patterns of thousands of stars. The interferometers point in different directions perpendicular to the common axis of rotation; this enables building up a rigid reference frame of positions and proper motions, as well as the determination of absolute parallaxes. The faint limiting magnitude allows a direct link to the extragalactic reference frame, making the stellar proper motions inertial. The required short-term stability of the interferometer baselines may be maintained by laser metrology, by means of beam combiners and passive control, or through a combination of both. Some of the CCDs will be equipped with colour filters for wideband (e.g. UBVRI) and intermediate-band photometry (e.g. Strömgren/Vilnius system) of all observed objects. The possibility to include a special radialvelocity instrument, aiming at a few $\mathrm{km} / \mathrm{s}$ accuracy at 15 th magnitude, is being considered. A more complete description of the GAIA concept is found in Lindegren \& Perryman (1997).

\section{Scientific Objectives}

Astrometric measurements provide model-independent estimates of basic geometric and kinematic properties of astronomical sources, in particular stellar distances and transverse velocities. The projected accuracy of $10 \mu$ as (microarcsec) for the positions and parallaxes, and $10 \mu \mathrm{as} / \mathrm{yr}$ for the 
proper motions, for stars down to $V \sim 15$, corresponds to a horizon of $\sim 10 \mathrm{kpc}$ for individual stellar distances and many times larger for statistical uses. The proper motion accuracy corresponds to transverse velocity errors of $1 \mathrm{~km} / \mathrm{s}$ at $20 \mathrm{kpc}$ (for known distances). Such astrometric accuracies (or better) would be obtained for tens of millions of stars to well-defined magnitude limits, and would be accompanied by accurate multi-colour, multi-epoch, sub-millimagnitude photometric measurements. The following is a brief summary of some important astrophysical applications of such data (for additional details, see Perryman et al. 1997).

Physics and Evolution of Stars. GAIA will provide extremely accurate ( $<1$ per cent) distances for stars out to $1 \mathrm{kpc}$ distance, encompassing the entire luminosity range from $M_{V}=-10$ to +20 , including all population types (also rare ones) represented in that volume, as well as numerous open clusters and associations. Detailed comparison with theoretical models will profoundly affect our understanding of stellar atmospheres, structure and evolution; indirectly, through the much improved determinations of stellar ages and chemical compositions, this will generate new information on the formation and evolution of the Galaxy.

Stellar Luminosities and the Distance Scale. The trigonometric parallaxes will provide direct and accurate luminosity calibration of an extremely wide variety of stellar objects, including evolutionary interesting objects such as massive stars, novae and planetary nebulae, and primary distance indicators such as Cepheids and RR Lyrae variables. For the first time this will establish an extensive network of distance measurements throughout a significant part of our Galaxy, including the central region, the bulge, spiral arms, and the halo. This network will even touch on the nearest companion galaxies, in particular the Magellanic Clouds, allowing the effects of the different formation histories of these systems to be studied.

Dynamics of Stellar Systems. At the sensitivity level of GAIA some 25 per cent of all stars will be resolved into components or otherwise manifest themselves as non-single (e.g. astrometric binaries). Many clusters, including a few globular clusters, will be available for detailed dynamical analysis based on the individual proper motions. Details of mass segregation, binary frequency, internal velocity structure, evaporation and tidal distortion can be studied for these objects.

Galactic Dynamics. The huge number of stars, the high accuracy for the parallaxes and absolute proper motions, and the faint limiting magnitude of GAIA would totally revolutionise dynamical studies of our Galaxy. Among the many questions that can be addressed with such data are: the nature of the spiral arms; the origin and history of the galactic bulge; the large-scale, threedimensional distribution of mass throughout the Galaxy, including dark matter in the disk and halo, and the total mass of the Galaxy; traces of galactic mergers and other important events in the history of the Galaxy, detectable by accurate mapping of a large volume of phase space.

Planetary Systems. GAIA will be able to screen hundreds of thousands of the bright nearby $(<100 \mathrm{pc})$ stars for possible astrometric signatures of planetary and brown dwarf companions. Jupiter-mass planets with periods up to 5 years can be detected out to some 50 pc and less massive companions to proportionally smaller distances.

General Relativity. From a global analysis of the light bending by the Sun, the PPN parameter $\gamma$, equal to unity in General Relativity, could be determined to about $10^{-6}$, a precision level where differences among alternative theories of gravitation may be discernible, and where higher-order effects of the solar system metric start to become apparent.

\section{References}

Lindegren, L. and Perryman, M.A.C. (1997) GAIA: Global Astrometric Interferometer for Astrophysics, Proc. HIPPARCOS Venice '97, ESA SP-402, pp. 799-802

Perryman, M.A.C., Lindegren, L. and Turon, C. (1997) The Scientific Goals of the GAIA Mission, Proc. HIPPARCOS Venice '97, ESA SP-402, pp. 743-748 